\title{
Nachrichten aus der Forschung
}

\section{Bastian Matteo Scianna \\ "Gewaltkulturen von den Kolonialkriegen bis zur Gegenwart.»}

\author{
Konferenz des Deutschen Historischen Instituts Warschau \\ mit dem Deutschen Komitee für die Geschichte des Zweiten \\ Weltkriegs in Kooperation mit der Universität Potsdam, \\ Potsdam, 4. bis 6. Juni 2015
}

DOI 10.1515/mgzs-2016-0009

Der interdisziplinäre Ansatz in der Gewaltforschung hat in den vergangenen Jahren mehrere Studien $\mathrm{zu}$ unterschiedlichen Kulturen der Gewalt hervorgebracht. Isabell Hulls Interpretation einer spezifischen deutschen »military culture« von den Kolonialkriegen bis zum Ersten Weltkrieg hat viele Anregungen geliefert, auch wenn ihre Thesen unlängst durch Alexander Watson angefochten wurden. Die Untersuchungen beziehen sich stets auf einen Gewaltraum, eine Situation oder auch die Organisation einer Armee, also einer sozialen Gruppe. Hieraus entsteht die Notwendigkeit, diese Beispiele vergleichend $\mathrm{zu}$ betrachten, um Ähnlichkeiten und Unterschiede nationaler Gewaltkulturen herauszuarbeiten und etwaige transnationale Trends beschreiben zu können.

Zahlreiche Fragen nach nationalen, zeitlichen, räumlichen und armeespezifischen Gewaltkulturen boten Anlass für die von Sönke Neitzel (London) und Stefan Lehnstaedt (Warschau) veranstaltete Konferenz. In den Beiträgen sollten jene nationalen Spezifika, aber auch über Ort, Zeit und Nation hinausreichende Interpretationsmodelle vorgestellt werden.

Die erste Sektion unter Vorsitz von Christoph Nübel (Berlin) befasste sich mit Kolonialgewalt vor dem Ersten Weltkrieg. Daqin Yang (Washington, DC) verdeutlichte das Paradox japanischer Herrschaft beispielhaft anhand des Boxeraufstandes. Auch wenn sich das Besatzungsregime in China ab 1937 äußerst brutal ausnehmen sollte, pries man die japanische Armee zu Beginn des 20. Jahrhunderts noch für ihre Disziplin. Wilde Plünderungen und die vereinzelte Exekution von Kriegsgefangenen seien zwar vorgekommen, riefen in der japanischen Presse aber Empörung hervor, woraufhin eine striktere Kontrolle eintrat. Yang bezog

Kontakt: Bastian Matteo Scianna, Universität Potsdam, E-Mail: scianna@uni-potsdam.de 
auch den russisch-japanischen Krieg mit ein, bei dem, trotz der Härte der Gefechte, die Kriegsgefangenen »cum grano salis« gut behandelt wurden. Eine Entwicklungslinie zu späteren Gewaltexzessen dürfe man, ohne die Auflösung des Bushido-Kodexes und insbesondere die situativen Faktoren abwägend zu betrachten, nicht ziehen.

Auch der zweite Beitrag von Timothy L. Schroer (Carrollton, Georgia, USA) befasste sich mit dem Boxeraufstand. Der Fokus lag auf der Interaktionsfunktion, die Gewalt einnehmen kann. Durch exemplarische öffentliche Gewalt habe das deutsche Expeditionskorps seinen eigenen Status vis-à-vis der chinesischen Bevölkerung und den anderen westlichen Armeen stärken wollen. Hiermit konnten laut Schroer transkulturelle Botschaften der Bestrafung und Erniedrigung - oder bei militärischen Paraden das Bild der disziplinierten Überlegenheit - vermittelt werden.

Am Beispiel der Jungtürken zwischen 1909-1912 zeigte James N. Tallon (Romeoville, Illinois, USA), wie zentralistischer Reform- und Fortschrittsglaube Aufstände hervorrief, deren Bekämpfung zur inneren Festigung der Modernisierung notwendig wurde. Zudem stellte Tallon dar, wie die militärischen Interventionen auch der äußeren Machtdemonstration dienten und den Zugriff auf Ressourcen sicherten. Auch wenn im Gebiet des heutigen Albanien, Syrien und Jemen oft exzessive Gewalt angewendet wurde, um die internen Rebellionen zu beenden, habe es beschränkende Faktoren gegeben, und oft seien auf Basis von Verhandlungen Kompromisse erzielt worden.

Christoph Nübel stellte resümierend fest, dass das "Zeitalter der Extreme» durch Modernisierungsbestrebungen und situative Exzesse schon vor 1914 begann. Instrumentalisierte Gewalt diente der Legitimierung in Kolonialräumen und zur Herstellung und Kommunikation neuer Hierarchien. Einschränkend wirkten soziale und religiöse Normvorstellungen der Soldaten sowie der disziplinarische Kodex.

Die zweite Sektion befasste sich mit Gewalt im Ersten Weltkrieg. Wolfram Dornik (Graz) schilderte die Gewalterfahrung von k.u.k. Soldaten an der Ostfront des Ersten Weltkrieges anhand von Tagebüchern und Memoiren. Er betonte die Vorerfahrungen mit Osteuropa als kulturellem Raum, welche die Soldaten bei passiv erfahrener sowie ausgeübter Gewalt beeinflussten. Ein Spezifikum der Gewalterfahrung sei die starke Involvierung von Zivilisten als Täter und/oder Opfer, der latente Antisemitismus, die Ideologisierung, aber auch die Abwesenheit von Gewalt gewesen.

Christoph Hertner (Bern) referierte zur Aufstandsbekämpfung durch die deutsche Armee in der Ukraine 1918. Er führte aus, wie unvorbereitet die Soldaten auf die Dynamiken des Gewaltraums waren und oft in harten Anfangsgefechten Gewalt als ordnendes Mittel einsetzten. Hertner betonte die situativen Aspekte 
und oft selbstständiges oder gar widersprüchliches Handeln der Truppenführer vor Ort. Nachdem die Truppe ihrem Orientierungsbedürfnis durch Gewalt nachgekommen war, konnte hiernach eine Entspannung einsetzen, da der Raum und die soziale Realität allmählich begreifbar wurden - beziehungsweise durch Gewalt neue Räume geschaffen worden waren.

Jens Boysen (Warschau) beleuchtete Polen als militärischen Akteur zwischen 1918 und 1948. Ein Hauptaugenmerk lag auf der Funktion des Opfernarrativs, das dazu diene, die eigene polnische Akteursfunktion auszublenden. Boysen hob hervor, dass in Polen ein stärkeres Bewusstsein dafür vorherrscht(e), wie eng Gewaltfähigkeit und Staatsfähigkeit zusammengehören. Daher sei Gewalt gegen Gruppen, die als anders wahrgenommen wurden, stets unter den Deckmantel des Staatsschutzes gefallen. Ähnlich wie im Beitrag von Wolfram Dornik zeigte sich auch hier, dass gerade die Generation, die die Kriegserfahrung verpasst hatte, in der Nachkriegszeit ihrem Wunsch nach Einsatz für die politische Sache oft radikal nachzukommen suchte. Die Grenzkonflikte hätten die Armee eine zu starke Stellung im Staat einnehmen lassen, und das polnische Sendungsbewusstsein, eine Ordnungsmacht in Osteuropa zu sein, habe ein Rapprochement mit den Nachbarstaaten verhindert.

In der Diskussion pointierte der Moderator Piotr Szlanta (Warschau) die Fremdheitserfahrung der Soldaten, sowie Gewaltausübung aufgrund militärischer Schwäche und als Legitimations- und Ordnungsmittel neuer Staatlichkeit. Gerade für Polen hob er das eminent wichtige Narrativ der Armee als Hauptträger der polnischen Staatsidee und erfolgreicher Kämpfer der Unabhängigkeit hervor. Eine Nachfrage zu den Unterschieden der Bayern und Österreicher in der Ukraine 1918 ergab, dass die deutschen Truppen eine Neigung zur Formalisierung des Ordnungswunsches hatten, obschon die Truppen der Donaumonarchie bessere Informationen über Bevölkerung und Raum besaßen. Ferner habe im Habsburger Heer kein ausschlagender Exzess wie etwa jener der 52. Württembergischen Landwehrbrigade 1918 in Taganrog stattgefunden.

Der Zweite Weltkrieg in Südeuropa war das von Peter Lieb (Potsdam) moderierte Thema der dritten Sektion. Tobias Hof (Chapel Hill, North Carolina, USA) beschäftigte sich mit der rassistischen Gewaltpolitik des faschistischen Italien und forderte eine stringentere Einbindung in die vergleichende Genozidforschung. Um diesem Anspruch gerecht zu werden, müsse man die Gewalterfahrung und kollektive Erinnerung aus den italienischen Kolonialkriegen berücksichtigen. An diesen Konflikten könne man bereits das destruktive Gewaltpotenzial der späteren Exzesse während der Besatzung des Balkans ablesen. Ein inhärentes Unterlegenheitsgefühl der »kleinen« Großmacht und vorherrschender Rassismus habe auch ohne faschistische Ideologie extreme Gewaltanwendung möglich gemacht. 
Federico Ciavattone (Pisa) analysierte die Aufstandsbekämpfung durch die Italienische Sozialrepublik (RSI) zwischen 1944 und 1945. Die Partisanenbekämpfung durch reguläre Armeeeinheiten fand in hunderten kleinen Operationen statt und traf auch die Bevölkerung und zivile Infrastruktur. Ciavattone zeigte allerdings, dass die Dimensionen der Aufstandsbekämpfung berücksichtigt werden müssen, und führte insgesamt 16 getötete Zivilisten und 206 tote Partisanen auf Aktionen der regulären RSI Armee zurück. Die Gewalteskalation im italienischen Bürgerkrieg zwischen 1943 und 1945 stelle also keine endlose Spirale der Gewalt in allen Bereichen dar.

Sabine Rutar (Augsburg) untersuchte den Gewaltraum Jugoslawien im Zweiten Weltkrieg auf regionale Unterschiede und »Sozialregionen«, um dem oft vorherrschenden Referenzrahmen der Ethnie entgegenzuwirken. In den untersuchten Bergbauregionen manifestierte sich Gewaltanwendung in Form von Zwangsrekrutierung, Deportationen und auch paramilitärischer Gewalt, denen Arbeiter insbesondere im Sozialraum der Minenarbeit entfliehen konnten.

Lieb fasste zusammen, dass man bei der Kategorisierung von Kriegsverbrechen stets den damaligen Referenzrahmen und Normenkatalog berücksichtigen müsse. Man könne erkennen, wie stark auch im italienischen Fall der Kolonialraum als »Experimentierfeld der Gewalt« diente, obgleich viele Fragen nach Gewalttransfers und Kontinuitäten weiterhin unerforscht blieben.

Die vierte Sektion unter dem Vorsitz von Takuma Melber (Mainz) widmete sich dem Zweiten Weltkrieg in Asien. Frank Jacob (New York) fokussierte sich auf die Frage, ob es einen spezifisch japanischen Zug der exzessiven Gewaltanwendung gegeben habe. Ausgehend von dem Nanking Massaker 1937/38, der Versklavung der »comfort women« und Behandlung der Kriegsgefangenen führte Jacob an, wie sich unorganisierte Brutalität in rechtsfreien Gewalträumen abspielte. Durch die Schaffung eigener moralischer und rechtlicher Normen sei die Gewaltspirale auch durch ein rassistisches Überlegenheitsgefühl und Dehumanisierung des Gegners verstärkt worden. Dies stelle keine japanische Ausnahme dar, sondern müsse vielmehr im Vergleich - etwa zum Deutschen Reich - gesehen werden.

Stephen R. MacKinnon (Tempe, Arizona, USA) untersuchte die Verknüpfung von Gewaltkulturen auf dem europäischen und asiatischen Kriegsschauplatz. Der Krieg in China sei von 1937-39 wiederholt mit dem spanischen Bürgerkrieg verglichen worden. Hinsichtlich Besatzungsherrschaft, Terror gegen die Zivilbevölkerung und Kollaborationsregime seien in Europa und Asien ähnliche Phänomene zu beobachten. Allerdings habe der Krieg in China nie ein wirkliches Ende gefunden. MacKinnon betonte, dass der Guerillakrieg taktisch und logistisch einen anderen Charakter vorweise und der Sieg Mao Zedongs bis heute grundlegend als Vorbild für asymmetrische Kriege bleibe. So sei dies die nachhaltigste 
Lehre aus dem Krieg, der an Rassismus und Brutalität, von allen kriegführenden Parteien, keine Ausnahme darstellte.

Zusammenfassend ging Melber auf die Charakteristika der japanischen Armee ein. Wie in der Zivilgesellschaft, herrschte hier ein Gruppendenken vor, welches das Individuum den Interessen des Ganzen unterordnete. Die Gruppendynamik habe daher energischer als in westlichen Armeen gewirkt und die Perversion des Bushdio-Kodex dazu geführt, dass selbst verbrecherische Befehle nie verweigert wurden. Die Omnipräsenz der Gewalt während der Ausbildung und die Radikalisierung des Offizierkorps in den 1930er Jahren erleichterte es zudem, Hemmschwellen abzubauen, auch wenn Detailstudien zu Schlüsselpersonen der Militärführung weiterhin fehlen.

Die fünfte Sektion betrachtete unter der Leitung von Gerhard P. Groß (Potsdam) die »Neuen Kriege« nach 1945. Marcel Berni (Zürich) ging auf die Zahlenund Wissenschaftsgläubigkeit der USA im Vietnamkrieg ein. Der »body count« habe den Versuch einer unvorbereiteten Armee dargestellt, mit neuesten wissenschaftlichen Managementmethoden auf den Guerillakrieg eine Antwort zu finden. Berni führte aus, dass bereits während des Krieges nur zwei Prozent der USGeneralität an den Erfolg dieser Vorgehensweise glaubte. Trotzdem kam es zu Gewaltexzessen, denen oftmals unschuldige Zivilisten zum Opfer fielen. Offensichtlich ging es darum, geforderte Quoten zu erfüllen. Diese »selbstevidente Identifizierung des Gegners« sei oft aus Frustration, Angst oder Ermutigung durch juristische Blankochecks erfolgt. Die strukturelle Gewaltspirale erzeugte zwar eine enorme Datensammlung, die aber nie ausgewertet wurde und somit keine neue militärische Lagebestimmung zur Folge hatte.

Robert Lučić (Potsdam) thematisierte die Transformation der Jugoslawischen Volksarmee auf den Schlachtfeldern Ostslawoniens 1991. Er beschrieb die Auflösung des staatlichen Gewaltmonopols anhand der Eroberung Vukovars. Hier kamen kulturelle Traditionen des Widerstandes und des Kleinkrieges zusammen, wie sie unter Tito existierten. Im Stadtkampf entwickelte sich eine situative Dynamik, die zu Gewaltexzessen führte. Die reguläre Armee war auf ortskundige Freischärler angewiesen, die aber oft persönliche Rechnungen beglichen und sich als schwer kontrollierbare Soldateska entpuppten.

Jan C. Behrens (Potsdam) sprach zur Genese der post-sowjetischen Gewaltkultur an der imperialen Peripherie. Der Afghanistankrieg stelle die eigentliche Zäsur der jüngeren russischen Geschichte dar, indem er »hobbesianische Räume« entstehen ließ und eine institutionelle Kontinuität der Gewaltapparate möglich machte. Behrens betonte die Gewalttransfers von Afghanistan nach Tschetschenien und teilweise in die heutigen Konfliktregionen der Ukraine. Gewalt gegen die Zivilbevölkerung, Rache und Vergeltung sei in Afghanistan zur Beherrschung des Gewaltraumes eingesetzt worden und habe neben dem Bedürfnis nach Selbst- 
erhaltung auch zur Bildung von Gewalt- und Solidargemeinschaften russischer Soldaten geführt. Dies habe längerfristige Folgen jenseits der situativen Gewaltausübung, die es historisch $\mathrm{zu}$ untersuchen gelte, um das Spannungsfeld zwischen Gewalt und Zivilität im post-sowjetischen Raum besser verstehen zu können.

Gerhard P. Groß resümierte, dass der Mangel an Kontrolle durch Offiziere, Institutionen oder die Gesellschaft $\mathrm{zu}$ einer Bestialisierung führe, was durch mangelnde Ausbildung der Truppen stets begünstigt würde. Zudem müsse man sich die Frage stellen, wie der Rückkehr von Gewalt(experten) aus der Peripherie, sowohl im ausgehenden 19. Jahrhundert als auch heute, zu begegnen sei. Alte oder neue Gewaltkulturen, so sie auch je nach Nation oder Heer unterschiedlich sein mögen, treffen jedenfalls auf gewaltlose post-heroische Gesellschaften im Westen.

Stephan Lehnstaedt zog ein Resümee und griff nochmals die Frage nach der Definition und dem Charakter von Gewalt auf: tolerierte, instrumentalisierte oder grauzonenartige Gewalt sowie die Abhängigkeit von Perspektive und Raum. Zudem bliebe unklar, wann, wenn überhaupt, die Erfahrung oder Wahrnehmung von Geschichte oder Gewalt sich $\mathrm{zu}$ einer »national culture« forme. Nationale Gewaltkulturen könnten somit als existent angesehen werden, etwa die der deutschen Wehrmacht im Ostkrieg, jedoch müsse die Abgrenzung strikt nach Ort und Zeit erfolgen. 